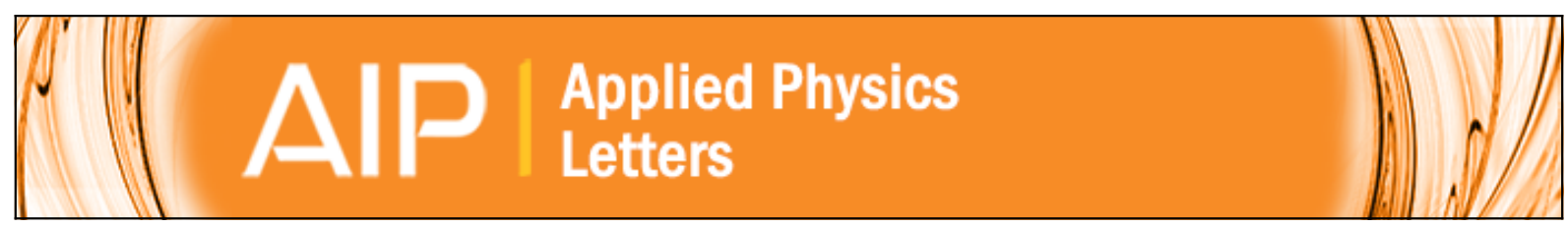

\title{
Ultrahigh dielectric constant of thin films obtained by electrostatic force microscopy and artificial neural networks
}

E. Castellano-Hernández and G. M. Sacha

Citation: Applied Physics Letters 100, 023101 (2012); doi: 10.1063/1.3675446

View online: http://dx.doi.org/10.1063/1.3675446

View Table of Contents: http://scitation.aip.org/content/aip/journal/apl/100/2?ver=pdfcov

Published by the AIP Publishing

\section{Articles you may be interested in}

Note: Artificial neural networks for the automated analysis of force map data in atomic force microscopy

Rev. Sci. Instrum. 85, 056104 (2014); 10.1063/1.4876485

Electron holography characterization of the electrostatic potential of thin high-K dielectric film embedded in gate stack

Appl. Phys. Lett. 99, 163506 (2011); 10.1063/1.3652770

Structural and dielectric study of parylene $C$ thin films

Appl. Phys. Lett. 94, 152901 (2009); 10.1063/1.3114404

Enhancement of dielectric constant in $\mathrm{Hf} \mathrm{O} 2$ thin films by the addition of $\mathrm{Al} 2 \mathrm{O} 3$

Appl. Phys. Lett. 89, 192905 (2006); 10.1063/1.2387126

Space-charge-limited leakage current characteristics influenced by field-dependent permittivity in high dielectric constant and ferroelectric thin films

Appl. Phys. Lett. 88, 212905 (2006); 10.1063/1.2203512

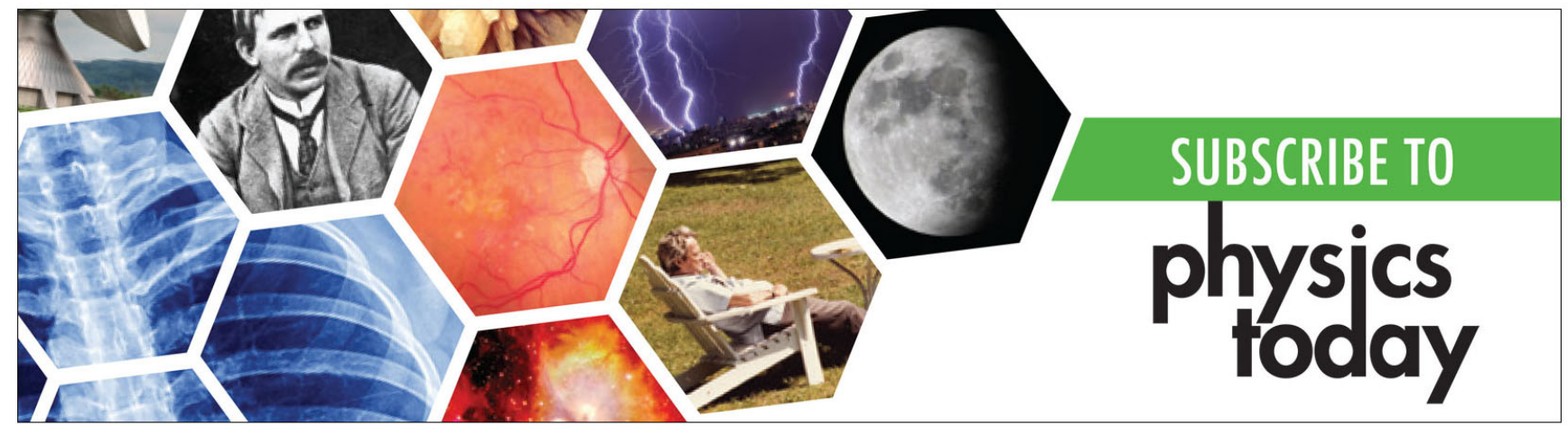




\title{
Ultrahigh dielectric constant of thin films obtained by electrostatic force microscopy and artificial neural networks
}

\author{
E. Castellano-Hernández and G. M. Sacha ${ }^{\text {a) }}$ \\ Departamento de Ingeniería Informática, Universidad Autónoma de Madrid, Campus de Cantoblanco, \\ 28049 Madrid, Spain
}

(Received 19 September 2011; accepted 14 December 2011; published online 9 January 2012)

\begin{abstract}
A detailed analysis of the electrostatic interaction between an electrostatic force microscope tip and a thin film is presented. By using artificial neural networks, an equivalent semiinfinite sample has been described as an excellent approximation to characterize the whole thin film sample. A useful analytical expression has been also developed. In the case of very small thin film thicknesses (around $1 \mathrm{~nm}$ ), the electric response of the material differs even for very high dielectric constants. This effect can be very important for thin materials where the finite size effect can be described by an ultrahigh thin film dielectric constant. (C) 2012 American Institute of Physics. [doi:10.1063/1.3675446]
\end{abstract}

Understanding the electric field effect in nanostructured thin films is a key issue in nanoscience nowadays. ${ }^{1}$ By applying a voltage between a force microscope tip and a sample, electrostatic force microscopy $(\mathrm{EFM})^{2-8}$ has been used to analyze different properties of thin films at the nanoscale. ${ }^{9}$ Recently, single and few layer graphene $(\mathrm{FLG})^{10}$ have attracted much attention because of its atypical response to electrostatic fields, which is in sharp contrast with that expected for conventional conducting or semiconducting films. ${ }^{11}$ To be applied in planar device architectures, FLG films must be placed on insulating surfaces. ${ }^{12}$ The effect of an insulating surface on the electronic structure of FLG has been theoretically studied before. ${ }^{13,14}$ Unfortunately, the effect of the electrostatic field under these restrictions has not been studied in detail since previous theoretical works focused on dielectric thin films over metallic substrates. ${ }^{15,16}$ In this article, we combine numerical methods and artificial neural networks ${ }^{17}$ (ANNs) to simulate the electrostatic interaction between an EFM tip and a thin film. Using the electrostatic force as input patterns to the ANN, we establish that the thin film sample can be replaced by a simple semiinfinite sample characterized by an effective dielectric constant. We show that, for typical EFM setups and thin film thicknesses around $1 \mathrm{~nm}$, the electrostatic interaction of thin films with ultrahigh dielectric constants is easily distinguishable. This effect must be taken into account when ultrathin materials such as FLG are being used.

In a typical EFM setup, we have a metallic tip connected to a battery that applies a constant electric potential $\mathrm{V}_{0}$. The tip is placed over a sample at a tip-sample distance D. The tip is characterized by three geometrical parameters: The apex radius $\mathrm{R}$, the half-angle $\theta$, and the length $\mathrm{L}$. To solve electrostatic problems with similar geometries, several numerical methods have been developed. ${ }^{18-21}$ Some software packages, based on the finite elements method, such as COMSOL or ELMER, have been also used to simulate electrostatic fields in EFM. ${ }^{15}$ In this article, we use the generalized image charge method $^{22,23}$ (GICM). The GICM replaces the surface charge

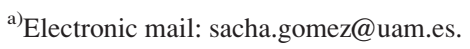

density by a set of charges inside the metallic tip and the sample by a set of image charges. The $\mathrm{q}_{\mathrm{i}}$ charge values are obtained by a standard least-squares minimization. Its efficiency has been demonstrated for systems where the sample includes objects such as Carbon Nanotubes ${ }^{24}$ or graphene. ${ }^{25}$

In the case of a thin film, the sample is composed by two layers: (1) a dielectric thin film with dielectric constant $\varepsilon_{1}$ and thickness $\mathrm{h}_{1}$ and (2) a dielectric substrate with dielectric constant $\varepsilon_{2}$. In Figure 1, we show equipotential distributions of a spherical tip over a thin film (top) and a semiinfinite dielectric sample (bottom). Once the charge distribution is obtained by the tip, the electrostatic force $\mathrm{F}$ and

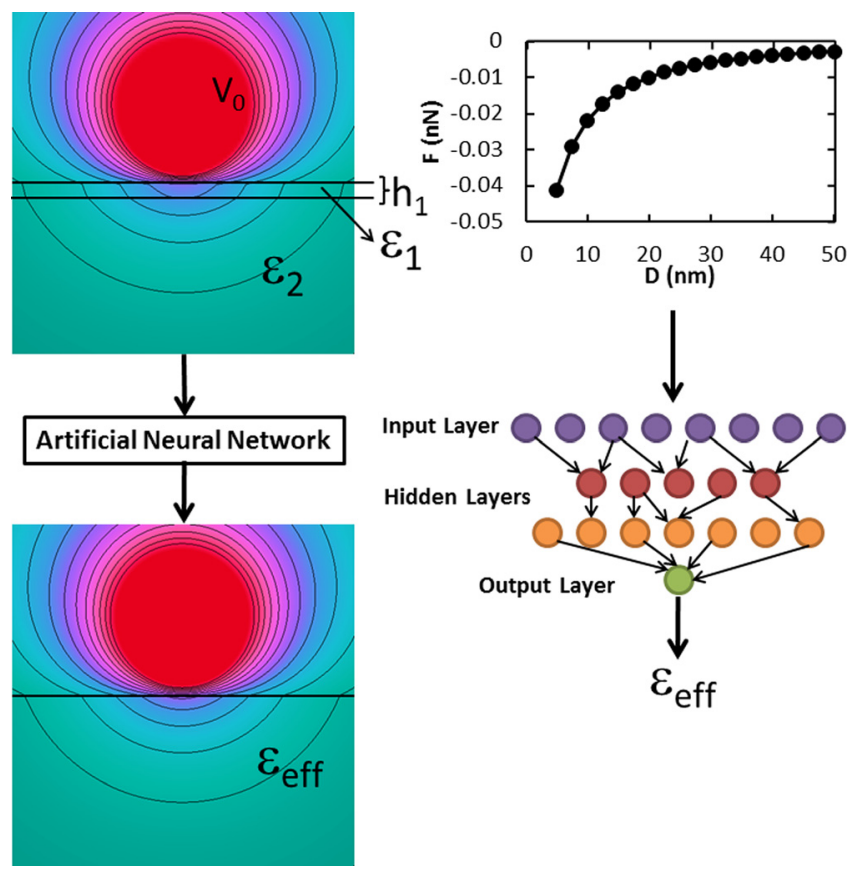

FIG. 1. (Color online) Scheme of the method to obtain the effective dielectric constant of a thin film sample. The electrostatic force of a system composed by a thin film over a dielectric substrate (equipotential lines shown at the top) is used as the input of an artificial neural network. The output value is the effective dielectric constant of an equivalent sample composed by a semiinfinite dielectric substrate (equipotential lines shown at the bottom). $\mathrm{R}=25 \mathrm{~nm}, \mathrm{~h}_{1}=5 \mathrm{~nm}, \varepsilon_{1}=10, \varepsilon_{2}=5, \varepsilon_{\text {eff }}=5.59, \mathrm{~V}_{0}=1 \mathrm{~V}$, and $\mathrm{D}=5 \mathrm{~nm}$. 
vertical force gradient $F^{\prime}$ can be directly obtained by the interaction between the tip charges and their images.

It has been well-established in the literature that the electrostatic interaction between tip and sample is a function of two magnitudes: the equivalent surface profile ESP (related to the sample geometry) and the response function RF (related to the microscope setup). ${ }^{26}$ To get a deeper understanding of the physical phenomena of our problem, we will try to find a simpler EFM geometry that produces the same interaction than the original problem. Our proposal is to replace the thin film by a semiinfinite dielectric sample characterized by an effective dielectric constant $\varepsilon_{\text {eff. This }}$ equivalent semiinfinite sample (ESS) can be easily calculated $^{23}$ and, although the ESS depends on the tip geometry (i.e., the RF), it can be easily related to the original thin film sample (i.e., ESP), which is independent of the EFM setup.

To obtain $\varepsilon_{\text {eff }}$, we use the artificial neural network described by Sacha et al. ${ }^{27}$ This ANN was used before to predict the dielectric constant of a semiinfinite dielectric substrate in an EFM system where the tip shape was unknown. In this article, we are going to use the F vs D curves of the thin film sample as inputs for the ANN. Since this ANN only has information about semiinfinite samples, the output value will be the dielectric constant of the semiinfinite sample that better reproduces the electrostatic force that was introduced to the ANN. In Figure 1 we show how the ANN establishes the relation between the thin film and its ESS. As an example, we have used a sample composed by a thin film $\left(\mathrm{h}_{1}=5 \mathrm{~nm}, \varepsilon_{1}=10\right)$ over a dielectric substrate $\left(\varepsilon_{2}=5\right)$ and a spherical tip $\left(\mathrm{R}=25 \mathrm{~nm}, \mathrm{~V}_{0}=1 \mathrm{~V}\right)$. In this case, the effective dielectric constant obtained by the ANN was $\varepsilon_{\text {eff }}=5.59$. In Figure 2(a), we show that the force gradient of the thin film and the ESS with $\varepsilon_{\text {eff }}=5.59$ are in striking agreement.

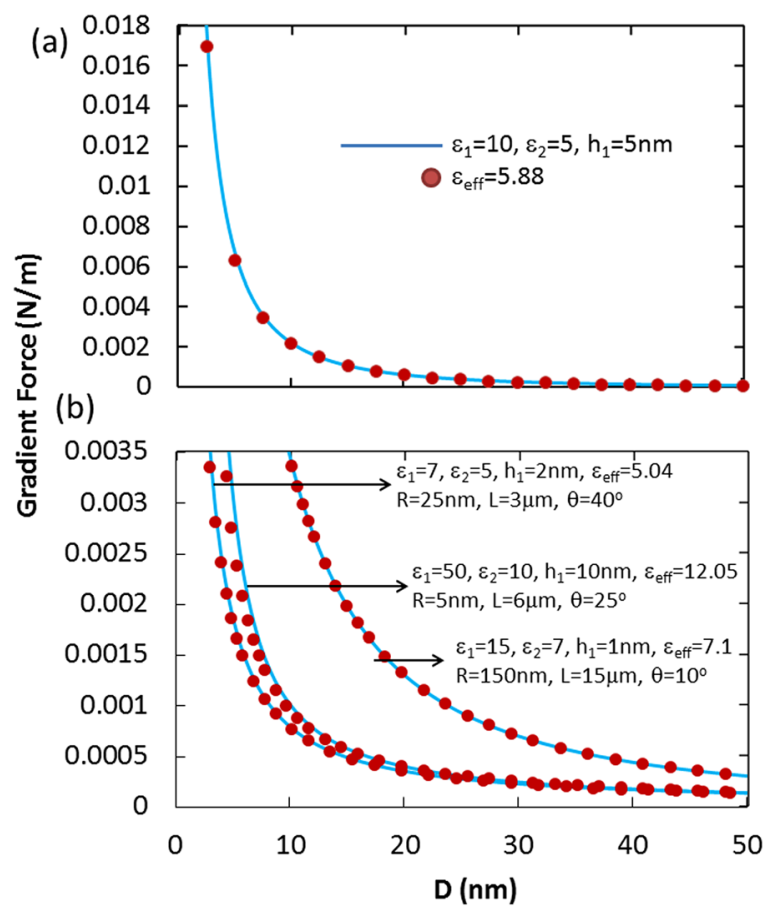

FIG. 2. (Color online) Gradient force versus tip sample distance for both a thin film and an equivalent semiinfinite dielectric sample obtained by artificial neural networks for a spherical tip with $\mathrm{R}=25 \mathrm{~nm}$ (a) and three different macroscopic tips (b). Details about the tip and sample geometries are shown in the figure.
Using the ANN, we only have to use the thin film F vs $\mathrm{D}$ curve as the input of the ANN, which gives a direct answer for the $\varepsilon_{\text {eff }}$, no matter what tip has been used. To obtain the best $\varepsilon_{\text {eff }}$, standard numerical methods need to compare the thin film F vs D curve with a set of ESS F vs D curves. This strategy needs much more calculations since the ESS F vs D curves must be independently calculated for every different tip used in the simulations. The ANN versatility allows us to obtain the $\varepsilon_{\text {eff }}$ for a wide variety of thin films, substrates and tips. We considered the following ranges: $\varepsilon_{1}=\{6,7, \ldots, 50\}, \varepsilon_{2}=\{5,6, \ldots, 50\}, \mathrm{h}_{1}=\{1,2, \ldots, 10\} \mathrm{nm}$ and $\mathrm{R}=\{25,50,100,200\} \mathrm{nm}$. We only included the cases where $\varepsilon_{1}>\varepsilon_{2}$. In general, the ESS reproduces the force gradient of the thin film with high accuracy (see Figure 2(a)). The maximum error obtained is smaller than $15 \%$. For the ranges described before, the relation between the thin film and the ESS follows a simple analytical expression given by

$$
C h_{1}=\left(\frac{\varepsilon_{e f f}-\varepsilon_{2}}{\varepsilon_{1}-\varepsilon_{e f f}}\right)^{A},
$$

where $\mathrm{C}$ is a constant which depends on the tip geometry $(\mathrm{C}=\mathrm{C}(\mathrm{R}, \theta, \mathrm{L}))$ and $\mathrm{A} \approx 3 / 2$ for all geometries analyzed. The constant $\mathrm{C}$ dependence with the tip geometry can be described by $\mathrm{C}=1 /(2 \mathrm{R})$ in the case of spherical tips with $25 \mathrm{~nm}<\mathrm{R}<200 \mathrm{~nm}$. It is worth noting that Eq. (1) gives adequate values for $h$ in the limits $\varepsilon_{\text {eff }}=\varepsilon_{1}\left(h_{1} \rightarrow \infty\right)$ and $\varepsilon_{\text {eff }}=\varepsilon_{2}\left(\mathrm{~h}_{1}=0\right)$. As the tip size increases, the dependence of $\mathrm{C}$ with the tip shape decreases. This is related to the fact that the electrostatic force dependence with the macroscopic shape of the tip decreases as the tip size increases. ${ }^{28}$ In the case of macroscopic tips, we obtained $\mathrm{C} \approx 0.00125 \mathrm{~nm}^{-1}$ for $\mathrm{L}=\{3,15\} \mu \mathrm{m}, \theta=\{10,40\}^{\circ}$, and $\mathrm{r}=\{5,200\} \mathrm{nm}$, which are typical values for EFM tips. This small value is in agreement with the tendency found before for spherical tips $C=1 /(2 \mathrm{R})$ since $\mathrm{C}$ becomes smaller as the tip size increases (i.e., big tips strongly feel the substrate below and minimizes the influence of the thin film). In Figure 3(b), we compare three different tips and thin film samples with their ESS, finding a striking agreement.

Let us now consider the electrostatic interaction for experimental setups with very small thin film thickness (i.e., $\mathrm{Ch}_{1} \rightarrow 0$ ). Within this limit, Eq. (1) can be written as

$$
\varepsilon_{e f f}=\left(C h_{1}\right)^{2 / 3} \varepsilon_{1}+\varepsilon_{2}\left[1-\left(C h_{1}\right)^{2 / 3}\right] .
$$

Within this approximation, $\varepsilon_{\text {eff }}$ increases linearly with both $\varepsilon_{1}$ and $\varepsilon_{2}$. However, their influence on $\varepsilon_{\text {eff }}$ is very different. In the case of the substrate dielectric constant, $\varepsilon_{\mathrm{eff}} / \varepsilon_{2} \approx 1$. On the other side, changes in $\varepsilon_{\text {eff }}$ due to $\varepsilon_{1}$ are proportional toCh ${ }_{1}{ }^{3 / 2}$. Taking into account that, for all the spherical tips, $\mathrm{Ch}_{1}{ }^{3 / 2}<0.1$ when $\mathrm{h}_{1}<=1 \mathrm{~nm}$, we find that $\varepsilon_{\text {eff }}$ changes at least 10 times slower than $\varepsilon_{1}$.

Now, we are going to demonstrate that this effect is even stronger when a macroscopic tip is included in the simulation. We have chosen a typical EFM tip characterized by $\mathrm{R}=25 \mathrm{~nm}, \mathrm{~L}=14 \mu \mathrm{m}$, and $\theta=17.5^{\circ}$. The sample is composed by a thin film characterized by $h_{1}=1 \mathrm{~nm}$ over a dielectric substrate with $\varepsilon_{2}=5$. As we can see in Figure 3(a), the extremely small value of $\mathrm{Ch}_{1}{ }^{3 / 2}$ implies that, even for 

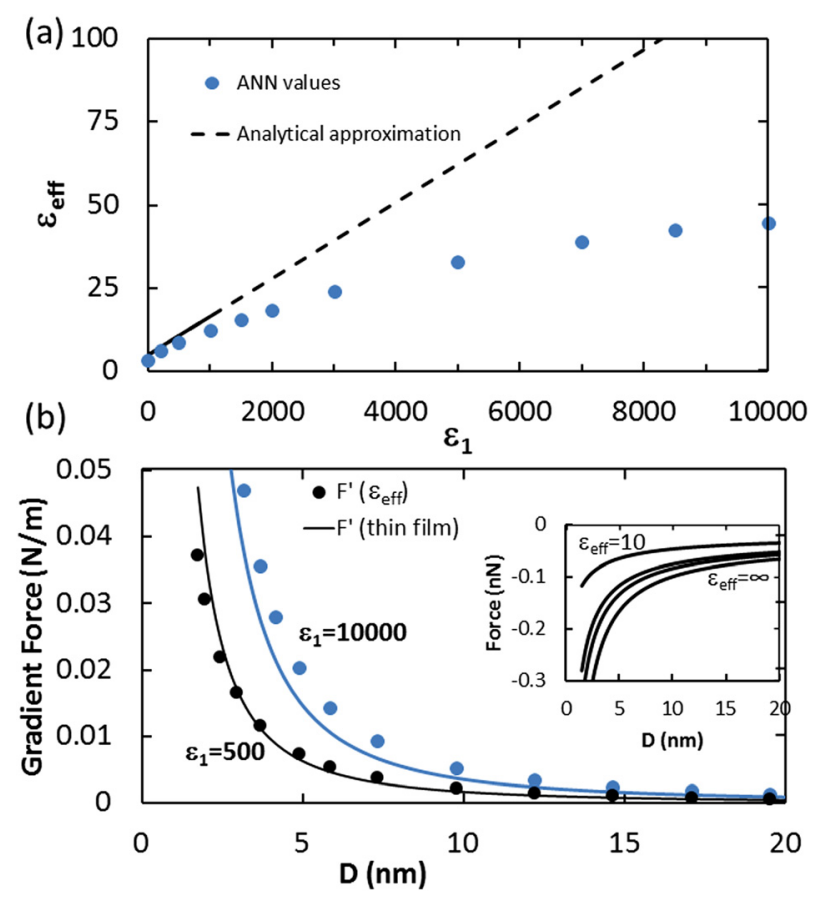

FIG. 3. (Color online) (a) Effective dielectric constant for ultrahigh thin film dielectric constants obtained by the analytical approximation and the ANN. Continuous line represents the range where the analytical expression gives better results than the ANN. (b) Gradient force for thin films characterized by $\varepsilon_{1}=500$ and $\varepsilon_{1}=10000$. Inset shows the electrostatic force for $\varepsilon_{\text {eff }}=10,30,50, \infty$ (top to bottom). In all figures, $R=25 \mathrm{~nm}, \theta=17.5^{\circ}$, $\mathrm{L}=14 \mu \mathrm{m}, \mathrm{V}_{0}=1 \mathrm{~V}, \mathrm{~h}_{1}=1 \mathrm{~nm}$, and $\varepsilon_{2}=5$.

$\varepsilon_{1}=10000, \varepsilon_{\mathrm{eff}}$ is still not bigger than $\varepsilon_{\mathrm{eff}}=50$. This implies that, for very small thin films, ultrahigh thin film dielectric constants $\varepsilon_{1}$ can be easily distinguished. For example, in Figure 3(b) we show simultaneously the force gradient for $\varepsilon_{1}=500$ and $\varepsilon_{1}=10000$. Even for these very high values, the two curves are easily distinguishable. In the inset of Figure 3(b), we show the electrostatic force for semiinfinite dielectric samples with $\varepsilon_{\text {eff }}$ between 10 and 50 , which are the ESS for the system under study in the range of $\varepsilon_{1}>1000$ (see Figure 3(a)). We can see that, even for the highest dielectric constant value $\varepsilon_{\text {eff }}=50$ (i.e., $\varepsilon_{1}=10000$ ), the electrostatic force is far from the metallic limit $\left(\varepsilon_{\text {eff }} \rightarrow \infty\right)$, which is approximately reached when $\varepsilon_{\text {eff }}>100$. This effect is due to the finite size of the thin film. Although the high dielectric constant induces a strong reduction of the electric field inside the material, its small size allows the electric field to reach the lower surface and penetrate inside the substrate.

In conclusion, we have demonstrated that, using artificial neural networks, the full structure of a thin film over a dielectric substrate can be replaced by an equivalent semiinfinite sample described only by an effective dielectric constant.
For thin film thicknesses around $1 \mathrm{~nm}$, we have demonstrated that thin film dielectric constants between 1000 and 10000 give very different electric responses. This effect could be of great interest in the study of thin materials with a high polarizability such as graphene layers since their finite size effects could be understood and characterized by an ultrahigh thin film dielectric constant.

Authors acknowledge J. J. Sáenz, P. Varona, and F. Rodríguez for insightful discussions. This work was supported by TIN2010-196079. G.M.S. acknowledges support from the Spanish Ramón y Cajal Program.

${ }^{1}$ E. M. Vogel, Nat. Nanotechnol. 2, 25 (2007).

${ }^{2}$ S. V. Kalinin, S. Jesse, B. J. Rodriguez, E. A. Eliseev, V. Gopalan, and A. N. Morozovska, Appl. Phys. Lett. 90, 212905 (2007).

${ }^{3}$ S. Guriyanova, D. S. Golovko, and E. Bonaccurso, Meas. Sci. Technol. 21, 025502 (2010).

${ }^{4}$ A. Verdaguer, M. Cardellach, J. J. Segura, G. M. Sacha, J. Moser, M. Zdrojek, A. Bachtold, and J. Fraxedas, Appl. Phys. Lett. 94, 233105 (2009).

${ }^{5}$ J. Hu, X.-D. Xiao, and M. Salmeron, Appl. Phys. Lett 67, 476 (1995).

${ }^{6}$ A. N. Morozovska, E. A. Eliseev, and S. V. Kalinin, J. Appl. Phys. 102, 074105 (2007).

${ }^{7}$ E. Palacios-Lidón, J. Abellán, J. Colchero, C. Munuera, and C. Ocal, Appl. Phys. Lett. 87, 154106 (2005).

${ }^{8}$ S. F. Lyuksyutov, R. A. Vaia, P. B. Paramonov, S. Juhl, L. Waterhouse, R. M. Ralich, G. Sigalov, and E. Sancaktar, Nature Mater. 2, 468 (2003).

${ }^{9}$ G. Gramse, I. Casuso, J. Toset, L. Fumagalli, and G. Gomila, Nanotechnology 20, 395702 (2009)

${ }^{10}$ K. S. Novoselov, A. K. Geim, S. V. Morozov, D. Jiang, Y. Zhang, S. V. Dubonos, I. V. Grigorieva, and A. A. Firsov, Science 306, 666 (2004).

${ }^{11}$ S. S. Datta, D. R. Strachan, E. J. Mele, and A. T. C. Johnson, Nano Lett. 9, 7 (2009).

${ }^{12}$ T. Burnett, R. Yakimova, and O. Kazakova, Nano Lett. 11, 2324 (2011)

${ }^{13}$ F. Guinea, Phys Rev. B 75, 235433 (2007).

${ }^{14}$ E. McCann, Phys. Rev. B 74, 161403 (2006).

${ }^{15}$ L. Fumagalli, G. Gramse, D. Esteban-Ferrer, M. A. Edwards, and G. Gomila, Appl. Phys. Lett. 96, 183107 (2010).

${ }^{16}$ I. Casuso, L. Fumagalli, G. Gomila, and E. Padrós, Appl. Phys. Lett. 91, 063111 (2007).

${ }^{17}$ S. Haykin, Neural Networks (Prentice-Hall, Englewood, NJ, 1999)

${ }^{18}$ M. Sausse-Lhernould, A. Delchambre, S. Regnier, and P. Lambert, Appl. Surf. Sci. 253, 6203 (2007)

${ }^{19}$ C. Riedel, A. Alegria, G. A. Schwartz, R. Arinero, J. Colmenero, and J. J. Saenz, Appl. Phys. Lett. 99, 023101 (2001).

${ }^{20}$ G. Gomila, J. Toset, and L. Fumagalli, J. Appl. Phys. 104, 024315 (2008).

${ }^{21}$ J. Colchero, A. Gil, and A. M. Baró, Phys. Rev. B 64, 245403 (2001).

${ }^{22}$ G. Mesa and J. J. Sáenz, Appl. Phys. Lett. 69, 1169 (1996)

${ }^{23}$ G. M. Sacha, F. Rodríguez, E. Serrano, and P. Varona, J. Electromagn. Waves Appl. 24, 1145 (2010).

${ }^{24}$ G. M. Sacha, C. Gómez-Navarro, J. J. Sáenz, and J. Gómez-Herrero, Appl. Phys. Lett. 89, 173122 (2006).

${ }^{25}$ G. M. Sacha, M. Cardellach, J. J. Segura, J. Moser, A. Bachtold, J. Fraxedas, and A. Verdaguer, Nanotechnology 20, 285704 (2009).

${ }^{26}$ S. Gómez-Moñivas, J. J. Sáenz, R. Carminati, and J. J. Greffet, Appl. Phys. Lett. 76, 2955 (2000).

${ }^{27}$ G. M. Sacha, F. B. Rodríguez, and P. Varona, Nanotechnology 20, 085702 (2009).

${ }^{28}$ S. Gómez-Moñivas, L. S. Froufe-Pérez, A. J. Caamaño, and J. J. Sáenz, Appl. Phys. Lett. 79, 4048 (2001). 